\title{
Estimation of Parasitic Resistance of Electrolytic Capacitor and Filter Inductor and Prediction of Input Filter Induced Oscillations in a Switch-Mode Magnet Power Supply
}

\author{
Rajul Lal Gour, ${ }^{1}$ Mangesh Borage, ${ }^{2}$ Alok Singh, ${ }^{2}$ and Sunil Tiwari ${ }^{2}$ \\ ${ }^{1}$ Department of Electrical Engineering, Jabalpur Engineering College, Jabalpur, Madhya Pradesh, India \\ ${ }^{2}$ Power Supplies and Industrial Accelerator Division, Raja Ramanna Centre for Advanced Technology, Indore, Madhya Pradesh, India \\ Correspondence should be addressed to Rajul Lal Gour; rajul1230@rediff.com
}

Received 30 June 2016; Revised 27 September 2016; Accepted 1 November 2016

Academic Editor: Don Mahinda Vilathgamuwa

Copyright (C) 2016 Rajul Lal Gour et al. This is an open access article distributed under the Creative Commons Attribution License, which permits unrestricted use, distribution, and reproduction in any medium, provided the original work is properly cited.

In switch-mode power converters with large ratings, it is important to be able to predict the parasitic resistances associated with circuit elements such as electrolytic capacitor and filter inductor in the initial converter design stage itself to avoid the cost and time associated with actual design, prototype fabrication, and testing of these components. Knowing the values of parasitic elements is also important as they decide the possibility of closed-loop instability, besides affecting the other circuit parameters. In this paper, a way to estimate the equivalent series resistance of electrolytic capacitor and the winding resistance of filter inductor is proposed leading to their closed form expressions in terms of system parameters. Using these, procedure to predict the closed-loop instability induced due to the input filter is exemplified with illustrative calculations.

\section{Introduction}

In particle accelerators, various magnets are used to bend, focus, and steer the beam of high energy charged particles such that the particles are maintained on the desired path and in the desired orbit [1]. These magnets, namely, dipole magnet, quadrupole magnet, sextupole magnet, and so forth, are mostly electromagnets in which the magnetic field produced is proportional to the current flowing in their coils. Therefore, a large number of current controlled power supplies are used to feed the coils of electromagnets. These magnet power supplies, apart from being output current controlled, have some special characteristics as compared to general purpose power supplies. Since the strength and quality of the magnetic field produced by the electromagnet depend on the current passing through it, the output current stability of the magnet power supply is required to be of the order of 10 to 1000 parts per million (ppm). The power supply is required to be operated in DC, slow ramped, or pulsed mode and sometimes required to track the set value. The load is inductive and since it is always connected in the circuit the load resistance variations are minor; small changes are induced only because of the change in operating temperatures.

A large number of topologies have been used to develop magnet power supplies. The choices of topologies depend on the output power rating and other operational requirements. Multipulse thyristor rectifiers [2], transistor series pass linear regulators with preregulators, switch-mode power supplies with high-frequency isolation transformer [3], and switchmode power converter with line frequency isolation [4-8] have been generally used. Every architecture has its own merits and demerits. The switch-mode power converter with line frequency isolation (called the chopper-type converter) is one of the most widely used topology mainly due to simplicity and ruggedness [4-8]. The block diagram of chopper-type converter is shown in Figure 1. The converter is fed through a rectifier which is followed by a second-order filter consisting of inductance $L_{f}$ and capacitance $C_{f}$. A three-phase transformer (not shown in the diagram) precedes the rectifier to step down the line voltage to match it with the required output DC voltage, $V_{o}$, and DC output current $I_{o}$ across the magnet load with the AC mains voltage. An insulated gate bipolar 


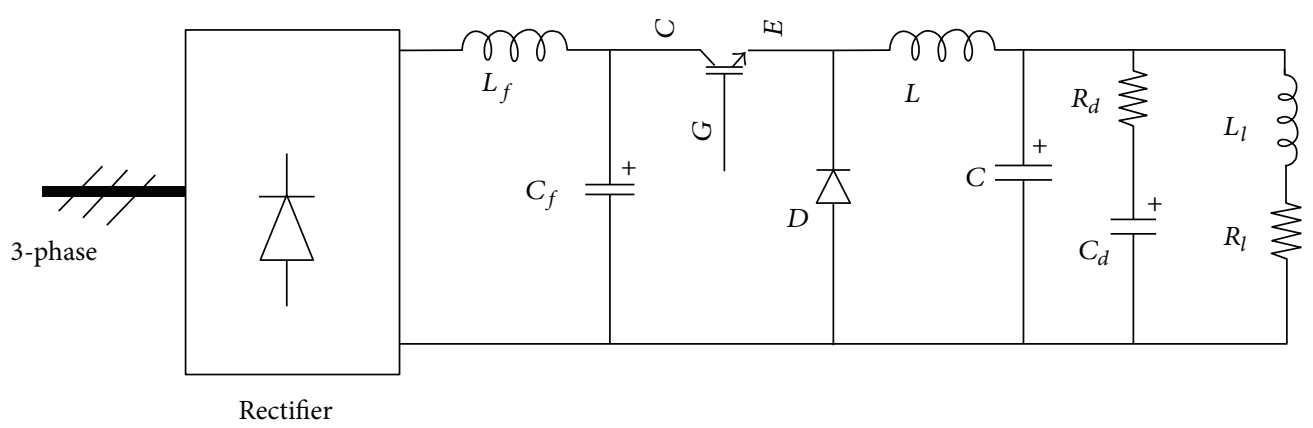

FIGURE 1: Architecture of chopper-type converter used as a magnet power supply.

transistor (IGBT) is used as the switching device. Another filter in the output consisting of inductance $L$ and capacitance $C$ attenuates the switching frequency ripple produced by the chopper stage. A damping branch with resistance $R_{d}$ and capacitance $C_{d}$ is added to the converter to damp the $L C$ filter. The magnet load is modelled by series combination of resistance $R_{l}$ and inductance $L_{l}$.

Since the input $L_{f} C_{f}$ filter contributes significantly to the overall size and weight of the converter, the conventional shunt capacitor damping [9] is not used to damp the filter resonance as the size of the damping capacitor and the overall size of the filter will increase. Instead, the designers often rely on the damping provided by the circuit parasitic components such as capacitors equivalent series resistor (ESR), winding resistance of the inductor, and the resistances offered by the interconnections and joints. It is well known that the resonant peak in the output impedance of the input filter in a switchmode converter can lead to the closed-loop oscillations if it becomes more than the input impedance of the switchmode converter [10]. In this context, it becomes important to investigate the sufficiency of the damping of the input filter offered by the parasitic resistors associated with it, before the converter is fabricated and tested. Besides, it is not uncommon to standardize the converter design for a group of magnets of different parameters to reduce the converter types and spares. During testing, the converter is sometimes tested with equivalent inductive load or even with the resistive load as the actual magnet and the power supply are developed almost simultaneously. Therefore, it would be useful to be able to predict the possibility of closed-loop instability under different loading conditions induced by the input filter at the design stage itself. To be able to achieve this, it would be necessary to predict the ESR of filter capacitor and the winding resistance of the filter inductance at the design stage itself, without necessitating carrying out the actual inductor design or its prototype fabrication. In this paper an attempt has been made to model the ESR of the capacitor in the form of curve-fit equation using the datasheet values of commercially available capacitors of two indicative makes. Further, a methodology to predict the winding resistance of the filter inductor using the various empirical relations given in [11] is explained leading to a closed form expression. Finally, the application of derived relationships to predict the stability of a converter is exemplified with illustrative calculations. A prototype magnet supply available in the laboratory that is based
TABLE 1: Parameters of the prototype supply.

\begin{tabular}{lc}
\hline Parameters & Values \\
\hline$I_{o}, V_{o}$ & $100 \mathrm{~A}, 20 \mathrm{~V}$ \\
Input ac mains & $415 \mathrm{~V}$, three-phase, $50 \mathrm{~Hz}$ \\
Transformer & $415: 23 \mathrm{~V}, \mathrm{DY} 11$ \\
$L_{f}, R_{s l}$ & $1 \mathrm{mH}, 2 \mathrm{~m} \Omega$ (measured) \\
$C_{f}, R_{s c}$ & $40 \mathrm{mF}, 4 \mathrm{~m} \Omega$ (estimated) \\
$L$ & $50 \mu \mathrm{H}$ \\
$C$ & $10 \mu \mathrm{F}$ \\
$R_{d}$ & $2 \Omega$ \\
$C_{d}$ & $20 \mu \mathrm{F}$ \\
$R_{l}$ & $0.2 \Omega$ \\
$L_{l}$ & Variable depending on \\
& actual magnet load, $2.6 \mathrm{mH}$ \\
\hline
\end{tabular}

on the architecture shown in Figure 1 has parameters listed in Table 1 which have been used to study the effect of these closed-loop oscillations induced by input filter in this paper.

\section{The Input Filter}

Design of filter inductor is done to maintain continuous inductor current with low ripple so as to improve the load regulation of the output voltage, reduce the harmonics in the input current, and improve the power factor [12]. The value of filter inductance $L_{f}$ is chosen to maintain continuous conduction mode till some value of the load current, called the critical current, $I_{\text {cric }}$ [12]. It can be obtained as follows:

$$
L_{f}=\frac{0.013 V_{l l}}{2 \pi f I_{\text {cric }}},
$$

where $V_{l l}$ is maximum line to line voltage and $f$ is line frequency in $\mathrm{Hz}$.

The value of filter capacitance $C_{f}$ is dependent on the cut-off frequency $f_{c}$ which is dependent upon the ripple attenuation as follows:

$$
\frac{V_{r}}{V_{\text {rect }}}=\left(\frac{f_{c}}{f_{\text {ripple }}}\right)^{2},
$$




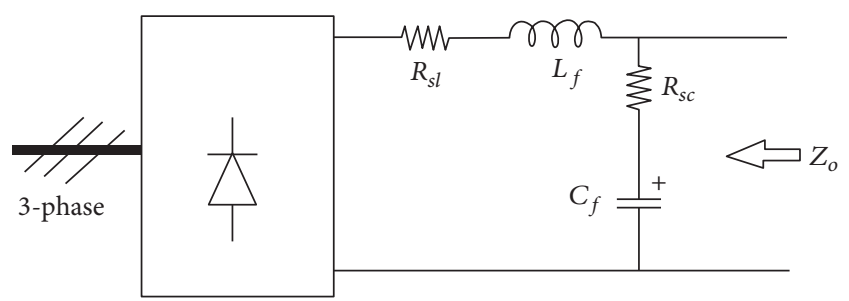

Rectifier

FIGURE 2: Input filter with parasitic resistances.

where $V_{r}$ is the ripple voltage at the output of $L_{f} C_{f}$ filter, $V_{\text {rect }}$ is the unfiltered rectifier voltage, and $f_{\text {ripple }}$ is the ripple frequency at the output of the rectifier. The ratio $\left(V_{r} / V_{\text {rect }}\right)$ or the attenuation required is governed by the allowable current in the magnet load at the ripple frequency. Having decided the value of filter cut-off frequency $f_{c}$, the value of filter capacitor is defined by

$$
C_{f}=\frac{1}{4 \pi^{2} f_{c}^{2} L_{f}} .
$$

Figure 2 shows the input filter section of the circuit shown in Figure 1. The ESR of capacitor, $R_{s c}$, and winding resistance of the inductor, $R_{s l}$, are explicitly shown. The output impedance $Z_{o}$ of the filter in terms of the filter components can be derived as follows:

$$
Z_{o}=\frac{L_{f} C_{f} R_{s c} s^{2}+\left(L_{f}+C_{f} R_{s c} R_{s l}\right) s+R_{s l}}{L_{f} C_{f} s^{2}+C_{f}\left(R_{s c}+R_{s l}\right) s+1} .
$$

A typical plot of (4) is shown in Figure 3 exhibiting peaking at the resonant frequency. The maximum output impedance, $Z_{o}(\max )$, can be derived as

$$
Z_{o}(\max )=\frac{L_{f}+C_{f} R_{s c} R_{s l}}{C_{f}\left(R_{s c}+R_{s l}\right)} .
$$

As the large value of output impedance of the input filter can deteriorate the audio susceptibility of the supply and cause the closed-loop oscillations under certain conditions, this filter has to be damped to reduce the peak resonance [9]. A common passive way of damping is to use shunt capacitor damping [9], which is generally used in the damping of the output $L C$ filter of the chopper as shown in Figure 1. The values of filter component are small, and the value and size of additional damping capacitor $C_{d}$ become practically manageable.

On the other hand, in case of the input filter, cut-off frequency is typically $20-30 \mathrm{~Hz}$ or lower if the output current stability specification of the power supply is stringent. Since the magnet power supplies are typically low-voltage highcurrent type, the value of filter capacitance is already large and it becomes practically difficult to provide shunt capacitor damping. The damping, therefore, is solely offered by the circuit parasitic components $R_{s c}$ and $R_{s l}$.
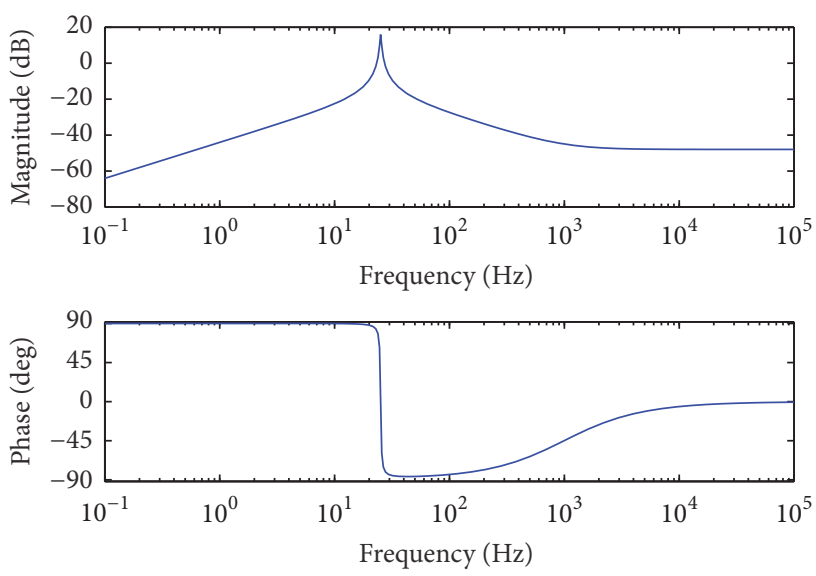

FIGURE 3: Plot of (4) showing the variation of output impedance of the input filter as a function of frequency $\left(L_{f}=1 \mathrm{mH} ; C_{f}=40 \mathrm{mF}\right.$; $R_{s c}=4 \mathrm{~m} \Omega ; R_{s l}=1 \mathrm{~m} \Omega$ ).

\section{Input Impedance of the Switch-Mode Converter}

It has been shown that a potential problem of closed-loop instability arises when the output impedance of the input filter becomes large than the input impedance of the switchmode converter. To study how this input filter affects the overall response of the system, the effect of the input filter impedance on the transfer function of the system has to be taken into account. This can be done using Middlebrook's extra element theorem [9]. According to which the modified transfer function of the system on addition of the extra element can be deduced by

$$
G^{\prime}(s)=G_{p}(s) \frac{\left(1+Z_{o}(s) / Z_{N}(s)\right)}{\left(1+Z_{o}(s) / Z_{D}(s)\right)}
$$

where $G^{\prime}(s)$ is the modified duty cycle to output transfer function of the converter with input filter and $G_{p}(s)$ is the duty cycle to output transfer function of converter without the input filter given by

$$
G_{p}(s)=\frac{V_{d}\left(a_{1} s+1\right)\left(a_{2} s+1\right)}{p_{1} s^{4}+p_{2} s^{3}+p_{3} s^{2}+p_{4} s+1},
$$

where

$$
\begin{aligned}
& a_{1}=C_{d} R_{d} \\
& a_{2}=\frac{L_{l}}{R_{l}} \\
& p_{1}=\frac{C L C_{d} R_{d} L_{l}}{R_{l}} \\
& p_{2}=\left(C L C_{d} R_{d}+\frac{C L L_{l}}{R_{l}}+\frac{L C_{d} L_{l}}{R_{l}}\right)
\end{aligned}
$$



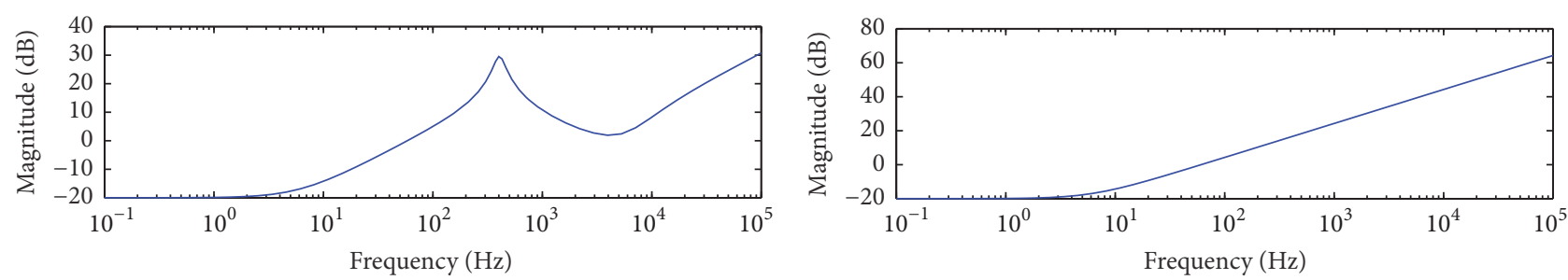

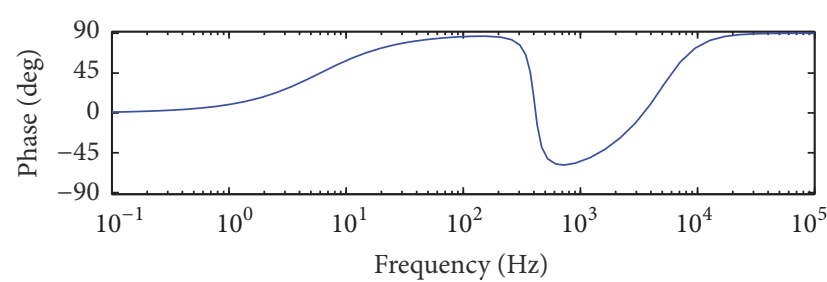

(a)

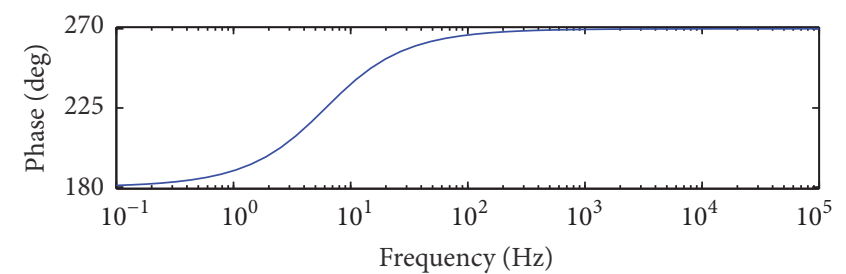

(b)

FIgURE 4: Plots of (9) and (11) showing (a) typical variation in $Z_{D}$ and (b) typical variation in $Z_{N}\left(L=50 \mu \mathrm{H} ; C=10 \mu \mathrm{F} ; C_{d}=50 \mu \mathrm{F}\right.$; $\left.R_{d}=2 \Omega ; L_{l}=2.6 \mathrm{mH} ; R_{l}=0.1 \Omega\right)$.

$$
\begin{aligned}
& p_{3}=\left(C L+\frac{L C_{d} R_{d}}{R_{l}}+L C_{d}+\frac{L_{l} C_{d} R_{d}}{R_{l}}\right) \\
& p_{4}=\left(\frac{L C_{d} R_{d}}{R_{l}}+\frac{L}{R_{l}}+\frac{L_{l}}{R_{l}}\right),
\end{aligned}
$$

with $L, C, C_{d}, R_{d}, L_{l}$, and $R_{l}$ being the converter parameters shown in Figure 1. (4).

$Z_{o}(s)$ is the output impedance of the input filter given by

$Z_{D}(s)$, also known as driving point impedance, is the Thevenin equivalent impedance seen from the converter input side with input set to zero [9]. For the converter shown in Figure 1, it can be derived as follows:

$$
Z_{D}(s)=\frac{1}{d^{2}}\left(\frac{A_{1} s^{4}+A_{2} s^{3}+A_{3} s^{2}+A_{4} s+R_{l}}{B_{1} s^{3}+B_{2} s^{2}+B_{3} s+1}\right),
$$

where $d$ is the duty cycle of the converter and,

$$
\begin{aligned}
& A_{1}=L L_{l} R_{d} C C_{d} \\
& A_{2}=\left(L L_{l} C_{d}+L R_{l} R_{d} C_{d} C+L L_{l} C\right) \\
& A_{3}=\left(C_{d} L_{l} R_{d}+C_{d} L R_{l}+C_{d} L R_{d}+C L R_{l}\right) \\
& A_{4}=\left(L+L_{l}+C_{d} R_{l} R_{d}\right) \\
& B_{1}=L_{l} R_{d} C_{d} C \\
& B_{2}=\left(L_{l} C_{d}+L_{l} C+R_{l} R_{d} C_{d} C\right) \\
& B_{3}=\left(R_{d} C_{d}+R_{l} C+R_{l} C_{d}\right) .
\end{aligned}
$$

$Z_{N}(s)$ is the impedance seen through the input port with input voltage nulled to zero [9]. For the converter shown in Figure 1, it can be derived as follows:

$$
Z_{N}(s)=-\frac{1}{d^{2}}\left(R_{l}+L_{l}(s)\right)
$$

Typical plots of (9) and (11) are shown in Figure 4. From (9) and (11) it can be inferred that both $Z_{D}(s)$ and $Z_{N}(s)$ are dependent on the duty cycle of the converter. The closed-loop instability occurs when output impedance of filter is greater than the input impedance of SMPS [10]. As the duty cycle of switch-mode converter is increased, $Z_{D}$ and $Z_{N}$ decrease. Therefore, the highest duty cycle $(d=1)$ is considered as the worst case for design. $Z_{D}$ and $Z_{N}$ also depend on the inductance and resistance values of the magnet load. Figure 5 shows the plots of $Z_{D}$ illustratively for different combinations of $R_{l}$ and $L_{l}\left(L_{l}=100 \mathrm{mH}, 2.6 \mathrm{mH}\right.$, and $\left.R_{l}=0.1 \Omega\right)$ superimposed on $Z_{o}\left(L_{f}=1 \mathrm{mH} ; C_{f}=40 \mathrm{mF} ; R_{s c}=4 \mathrm{~m} \Omega\right.$; $R_{s l}=1 \mathrm{~m} \Omega$ ). The graphs with different values of load inductance are shown to emphasize the effect of load inductance on stability. From the plots it can be observed that a high inductive load ensures the stability of the regulator because the high inductance offers high input impedance even at the low frequencies. However, with loads with lower inductance and the pure resistive load, the system may become unstable. Therefore, the resistive load case at the maximum duty cycle becomes the worst case conditions as far as the possibility of input filter induced oscillations is concerned. Thus the condition for stability can be stated as follows:

$$
Z_{o}(\max ) \leq R_{l} \text {. }
$$

Since the value of $Z_{o}(\max )$ given by (5) depends also on $R_{s l}$ and $R_{s c}$ besides the values of $L_{f}$ and $C_{f}$, it would be necessary to be able to predict $R_{s l}$ and $R_{s c}$ at the design stage itself, without necessitating to carry out the actual inductor design or its prototype fabrication.

\section{Estimation of Capacitor ESR}

Electrolytic capacitors are commonly used as the filter capacitor in the front end mains rectifier circuits. In this section an attempt to model the ESR of filter capacitor is presented based on the datasheet values of commercially available capacitors. ESR of a capacitor is the sum of resistance of dielectric, plate 

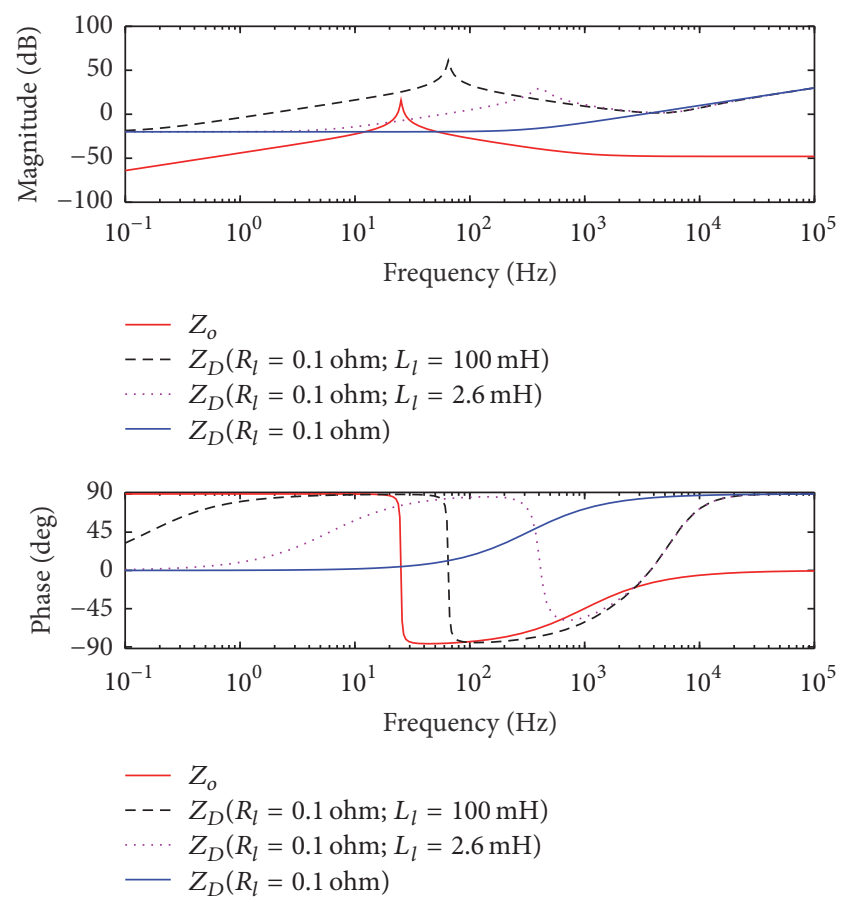

FIGURE 5: Impedance of regulator with inductive and resistive load $\left(L=50 \mu \mathrm{H} ; C=10 \mu \mathrm{F} ; C_{d}=50 \mu \mathrm{F} ; R_{d}=2 \Omega ; L_{l}=100 \mathrm{mH}\right.$, $2.6 \mathrm{mH} ; R_{l}=0.1 \Omega$ ).

material, electrolytic solution, and lead terminals. During the design of the filter the ESR values determine how much AC ripple current can the capacitor withstand. The ESR of a capacitor also depends on its value, voltage rating, maximum datasheet temperature, type of construction, ripple current rating, manufacturer, and so forth. Therefore, perhaps it is difficult to obtain a unique relationship that would describe the ESR of a capacitor as a function of its value alone.

The relationship between ESR and the capacitance value is studied by analyzing datasheet values of various capacitors of two representative makes (Make- $X$ and Make- $Y$ ) rated for $100 \mathrm{~V}, 200 \mathrm{~V}, 450 \mathrm{~V}$, and $85^{\circ} \mathrm{C}$ with screw terminals. Firstly, the datasheet values of ESR of the individual capacitors are plotted as a function of capacitance value for different voltage ratings (as shown by the $\triangle$ and $\bigcirc$ markers in Figure 6). Secondly, it is a common practice to use capacitor banks in which various capacitors are connected in parallel either because a single capacitor with required value, voltage rating, and ripple current rating is not available or to increase the ripple current rating or to reduce ESR. Therefore, the capacitor banks, in which maximum up to 10 identical capacitors are connected in parallel, are also considered. The calculated effective ESR value of such banks is also plotted as a function of effective capacitance (as shown by $\square$ and $\diamond$ in Figure 6). In principle, the capacitor banks in which capacitors are connected in series can also be considered for data generation. However, such capacitor banks are not commonly encountered since a magnet power supply is typically a low-voltage high-current type and therefore not considered in Figure 6. The results are
TABle 2: Values of coefficients $A$ and $B$.

\begin{tabular}{lcc}
\hline Capacitor voltage rating & $A$ & $B$ \\
\hline $100 \mathrm{~V}$ & $3.1 \times 10^{-4}$ & 0.79 \\
$200 \mathrm{~V}$ & $3.509 \times 10^{-4}$ & 0.816 \\
$450 \mathrm{~V}$ & $3.864 \times 10^{-4}$ & 0.769 \\
\hline
\end{tabular}

summarized in Figure 6 for 100 V, 200 V, and 450 V, screw terminal electrolytic capacitors rated for $85^{\circ} \mathrm{C}$.

From Figure 6 it can be observed that the relationship between $R_{s c}$ and $C_{f}$ can be expressed in following general form:

$$
R_{s c}=A C_{f}^{(-B)},
$$

where coefficients $A$ and $B$ for different voltage ratings of electrolytic capacitors of different voltage ratings are tabulated in Table 2. By following the general method described above one can obtain the relationship between ESR and the capacitance value for capacitors of the required voltage rating.

The ESR $R_{s c}$ can be written in terms of system parameters from (1), (3), and (13):

$$
R_{s c}=A\left(\frac{37.035 f I_{\text {cric }}}{\pi V_{l l} f_{c}^{2}}\right)^{-B}
$$

which further can be alternatively arranged as follows:

$$
R_{s c}=A\left(\frac{50 f\left(I_{\text {cric }} / I_{o}\right)}{\pi f_{c}^{2}}\right)^{-B}\left(R_{l}\right)^{B}
$$

where $I_{o}$ is the rated output current. Thus, the ESR of the filter capacitor can be predicted from the above relationships.

\section{Estimation of Inductor's Winding Resistance}

Various empirical relationships that can be used to predict the volume and surface area of an inductor are given in [11] based on function of many parameters such as inductance value, current, operating flux density, current density, temperature rise, and core configuration. Using these relationships, the following derivation is proposed to predict the winding resistance of an inductor at the design stage itself using various system parameters and without having to design the actual inductor or to prototype it.

The value of input filter inductor $L_{f}$ is fixed based on (1). The total energy stored by the inductor is related to the area product $A_{p}$ given by [11]

$$
A_{p}=\left(\frac{2 E \times 10^{4}}{k_{u} k_{j} B_{m}}\right)^{1.14},
$$

where $B_{m}$ is the maximum flux density (just before saturation) and $k_{j}$ is a constant related to core configuration [11]. $E$ is energy stored in inductor and $k_{u}$ is window utilization factor.

Energy stored in inductor is given by

$$
E=\frac{1}{2} L_{f} I_{o}^{2}
$$




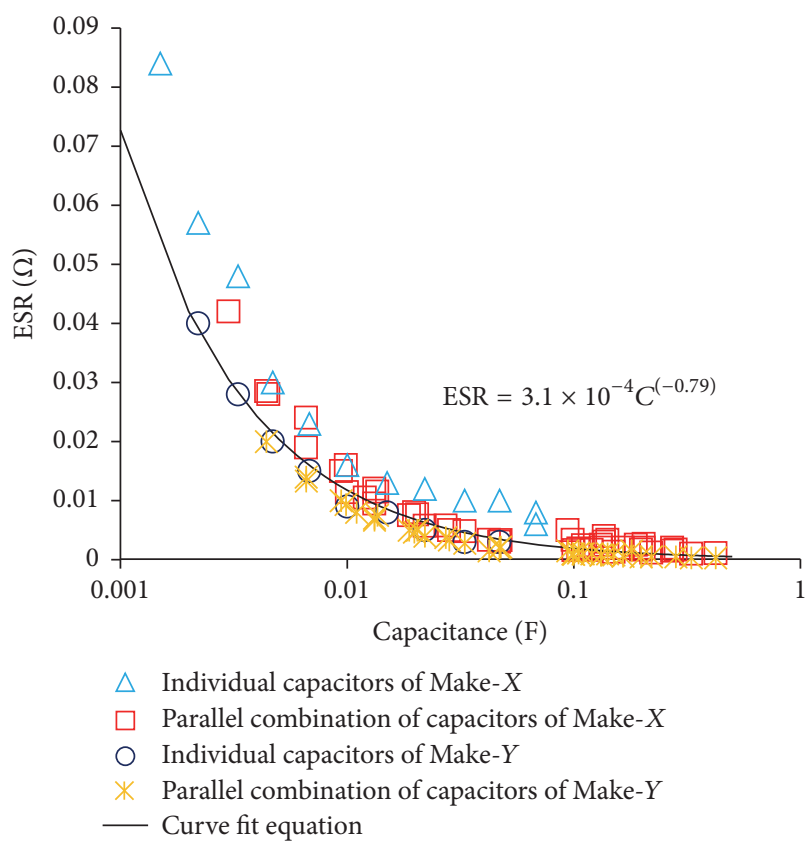

(a)

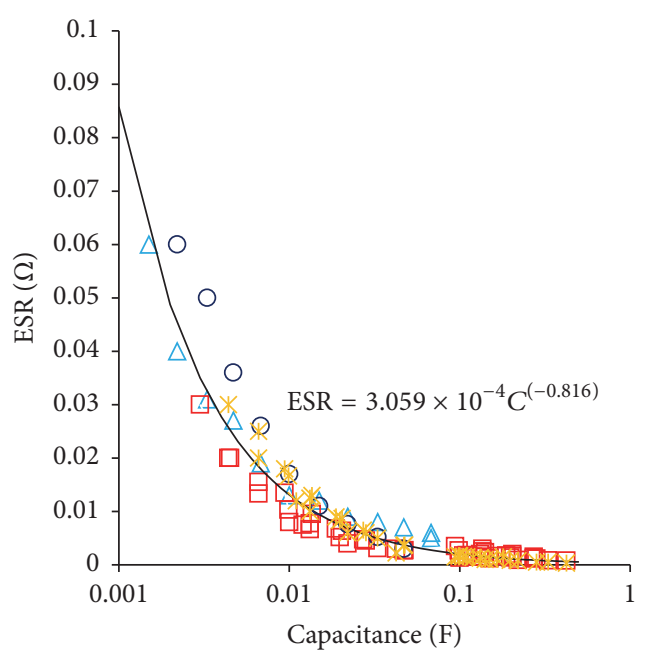

$\triangle \quad$ Individual capacitors of Make- $X$

$\square$ Parallel combination of capacitors of Make- $X$

O Individual capacitors of Make- $Y$

* Parallel combination of capacitors of Make- $Y$

— Curve fit equation

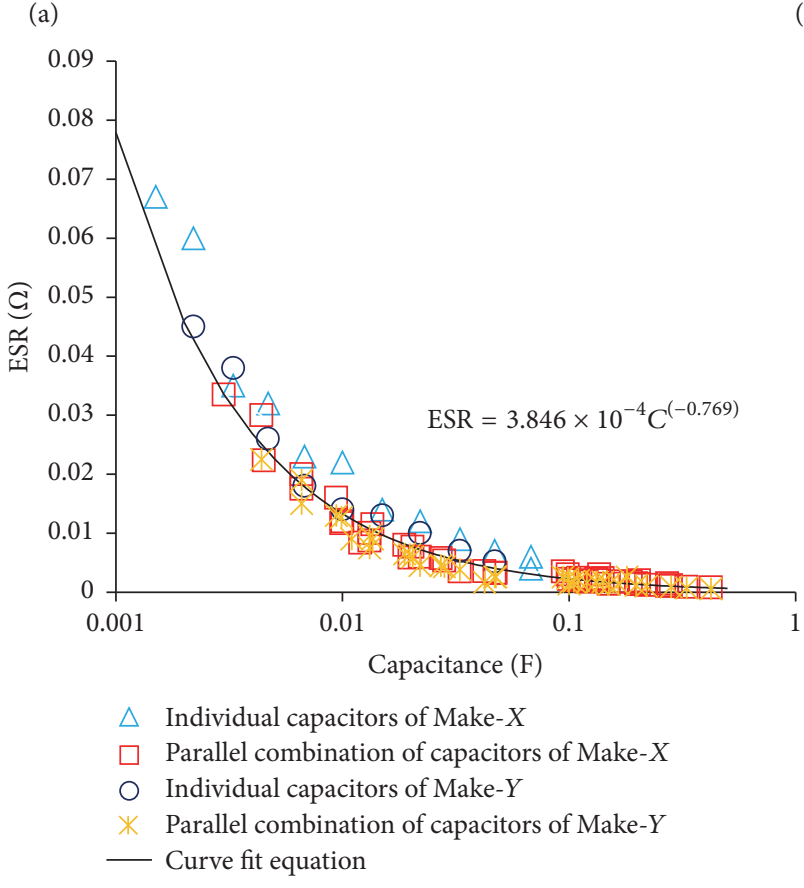

(b)

(c)

FIGURE 6: Relationship between ESR and capacitance value of electrolytic capacitors obtained for (a) $100 \mathrm{~V}$ (b) $200 \mathrm{~V}$ and (c) $450 \mathrm{~V}$, screw terminal electrolytic capacitors rated for $85^{\circ} \mathrm{C}$.

Substituting the value of $L_{f}$ from (1) in (17) we get

$$
E=\frac{1}{2}\left(\frac{0.013 V_{l l} I_{o}^{2}}{2 \pi f I_{\text {cric }}}\right) .
$$

Now substituting the value of $E$ from (18) in (16) we get

$$
A_{p}=\left(\frac{130 V_{l l} I_{o}^{2}}{2 \pi f I_{\text {cric }} k_{u} k_{j} B_{m}}\right)^{1.14} \text {. }
$$

During operation, due to the presence of parasitic winding resistance $R_{s l}$, some energy is lost in the form of heat. Due to these copper losses the temperature of the inductor increases. The rise in temperature, $\Delta T$, of surface area, $A_{t}$, is related to the copper losses incurred by the inductor as follows:

$$
I_{o}^{2} R_{s l}=A_{t} \psi
$$


where $\psi$ is temperature constant given by

$$
\Delta T=450(\psi)^{0.826} \text {. }
$$

Typically, $\psi=0.07$ for $\Delta T=50^{\circ}$ and $\psi=0.03$ for $\Delta T=25^{\circ} \mathrm{C}$.

Further the surface area $A_{t}$ of the inductor is also related to the area product as [11]

$$
A_{t}=k_{s} \sqrt{A_{p}}
$$

where $k_{s}$ is a constant dependent on the core configuration. The value of $k_{s}$ and $k_{j}$ for different types of core is given in [11].

Substituting value of $A_{p}$ from (19) in (22) we get

$$
A_{t}=k_{s}\left(\frac{130 V_{l l} I_{o}^{2}}{2 \pi f I_{\mathrm{cric}} k_{u} k_{j} B_{m}}\right)^{0.57} .
$$

And from (20) and (23) we get

$$
I_{o}^{2} R_{s l}=\psi k_{s}\left(\frac{130 V_{l l} I_{o}^{2}}{2 \pi f I_{\text {cric }} k_{u} k_{j} B_{m}}\right)^{0.57} .
$$

Hence the winding resistance $R_{s l}$ of the filter inductor in terms of the system parameters can be given as follows:

$$
R_{s l}=\psi k_{s}\left(I_{o}\right)^{(-0.86)}\left(\frac{130 V_{l l}}{2 \pi f k_{u} k_{j} B_{m} I_{\text {cric }}}\right)^{0.57}
$$

The above equation can be alternatively written in terms of rated output power $P_{o}$ and rated output current $I_{o}$ as

$$
R_{s l}=\psi k_{s}\left(I_{o}\right)^{(-2)}\left(\frac{100 P_{o}}{2 \pi f k_{u} k_{j} B_{m}\left(I_{\text {cric }} / I_{o}\right)}\right)^{0.57} .
$$

\section{Predicting Possibility of Closed-Loop Oscillations}

The previous sections deal with the estimation of parasitic resistors associated with electrolytic capacitor and filter inductor. These parasitic resistors, along with other parasitic resistors such as those of interconnecting wires and bus bars and joints, offer damping to the filter. The other parasitic resistance due to interconnections and joints can be neglected safely as they will offer additional damping. If the damping offered by $R_{s c}$ and $R_{s l}$ is sufficient to satisfy inequality of (12) the closed-loop system will be stable; else the input filter can induce closed-loop oscillations. This is exemplified with illustrative calculations in this section for a converter whose major parameters are listed in Tables 3 and 1. For the design of input filter, the ratio $\left(I_{\text {cric }} / I_{o}\right)$ is used as an independent variable. Electrolytic capacitors of $100 \mathrm{~V}$ rating are considered for estimation. The calculations are done using a spreadsheet program with the following steps:

(i) Calculate $L_{f}$ using (1).

(ii) Calculate $C_{f}$ using (3).

(iii) Calculate $R_{s l}$ using (25).

(iv) Calculate $R_{s c}$ using (14).
TABLE 3: Parameters of converter for illustrative calculations.

\begin{tabular}{lc}
\hline Parameters & Values \\
\hline$V_{l l}$ & $23 \mathrm{~V}$ \\
$f$ & $50 \mathrm{~Hz}$ \\
$f_{c}$ & $25 \mathrm{~Hz}$ \\
$B_{m}$ & $1 \mathrm{~T}$ \\
$k_{j}$ & $366[11]$ \\
$k_{s}$ & $39.2[11]$ \\
$k_{u}$ & $0.4[11]$ \\
$\psi$ & $0.03[11]$ \\
$I_{o}$ & $100 \mathrm{~A}$ \\
$V_{o}$ & $20 \mathrm{~V}$ \\
\hline
\end{tabular}

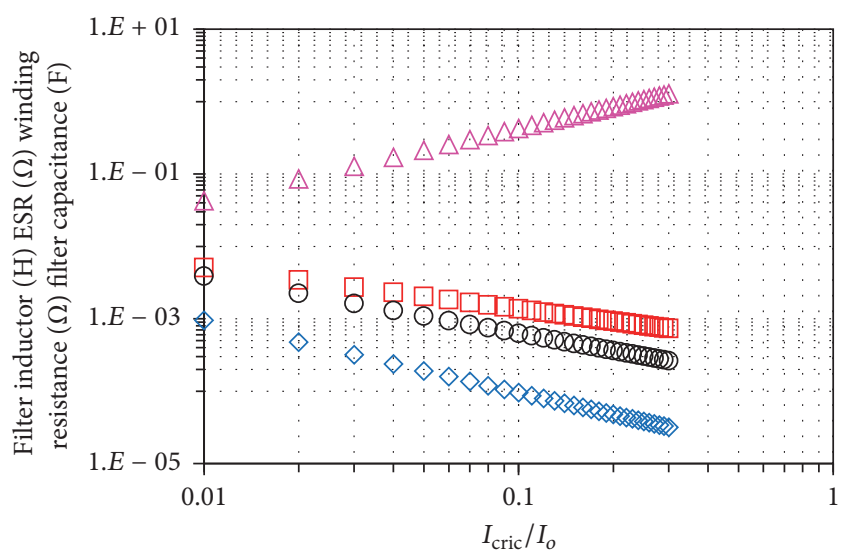

$\square$ Winding resistance of inductor (ohm)

$\diamond$ Filter inductance Lf $(\mathrm{H})$

$\bigcirc$ ESR of capacitor (ohm)

$\triangle$ Filter capacitance $\mathrm{Cf}(\mathrm{F})$

FIGURE 7: Variation of filter parameters with $I_{\text {cric }}$.

The results of the calculations are summarized in Figure 7. From Figure 7 it can be observed that as the value of critical inductor current $I_{\text {cric }}$ increases the value of filter capacitance increases as it is directly proportional to $I_{\text {cric }}$, whereas value of filter inductor and the parasitic resistances $R_{s l}$ and $R_{s c}$ decrease. This is due to the inverse relation between these parameters and $I_{\text {cric }}$. Next, the value of $Z_{o}(\max )$ is calculated from (5) and summarized in Figure 8. It can be observed that as the value of critical current $I_{\text {cric }}$ increases the output impedance of the system decreases. The critical condition arises when $Z_{o}(\max )$ becomes equal to the resistance as given by (12). In the present case, the converter will be stable if $I_{\text {cric }} / I_{o}$ is chosen to be more than 0.07 . To verify the results of Figure 8, two illustrative operating points in the unstable region (case I: $I_{\text {cric }} / I_{o}=0.02$ ) and the stable region (case II: $\left.I_{\text {cric }} / I_{o}=0.1\right)$ are selected. Calculated values of the various parameters are listed in Table 4. The plots of control-tooutput transfer function calculated using (6)-(11) and putting parameters from Tables 1 and 4 for these two cases are shown in Figure 9. It can be clearly seen that the system is unstable in case I and stable in case II. 


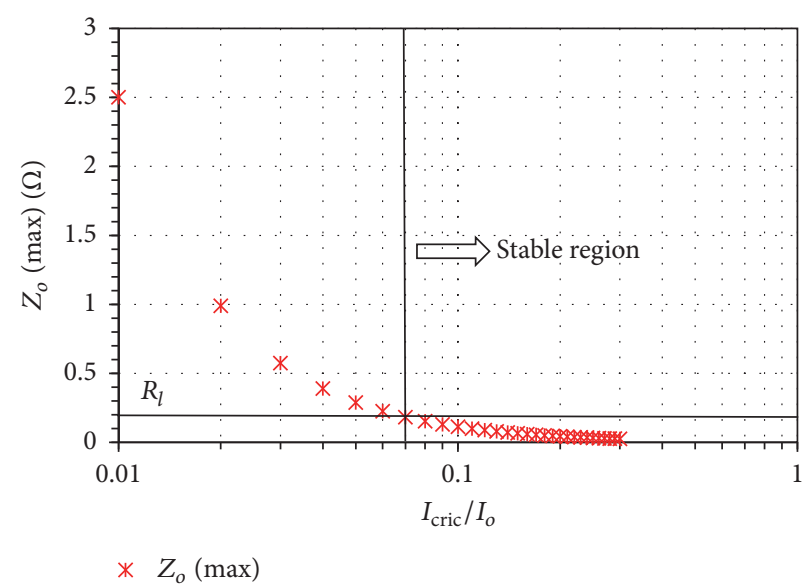

Figure 8: Variation of $Z_{o}(\max )$ with $I_{\text {cric }}$.
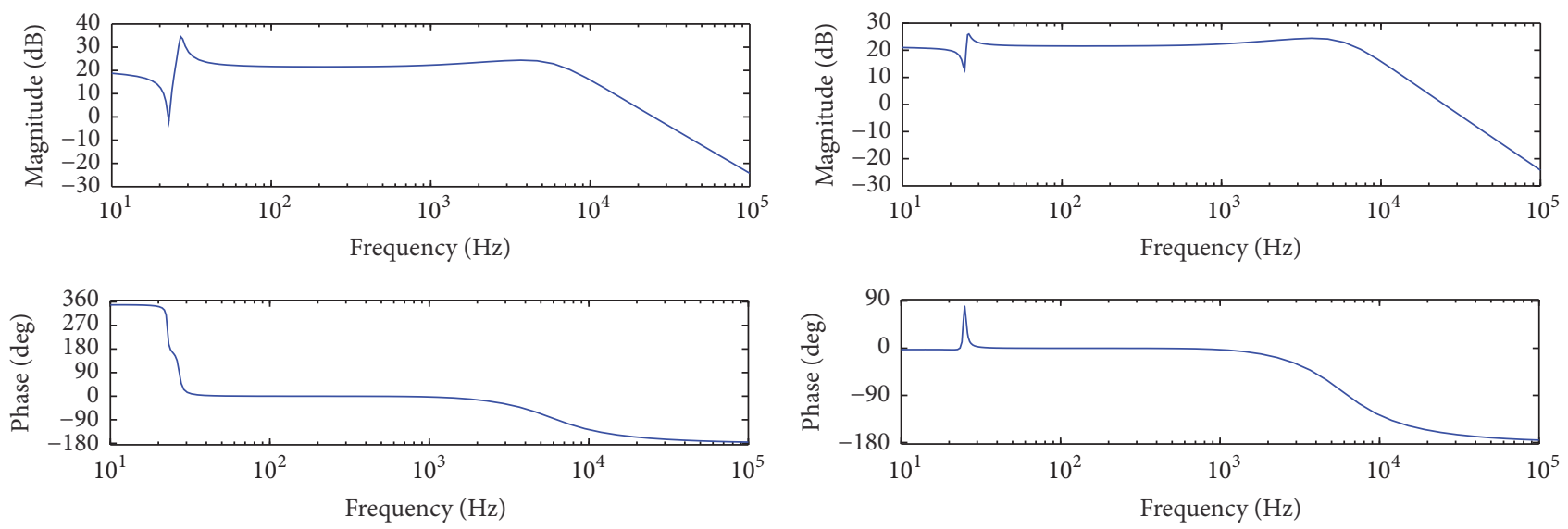

(a)

(b)

FIgURE 9: Control-to-output transfer function of in (a) case I and (b) case II.

TABLE 4: Parameters for calculation of control-to-output transfer function.

\begin{tabular}{lcc}
\hline Parameter & Case I & Case II \\
\hline$I_{\text {cric }} / I_{o}$ & 0.02 & 0.1 \\
$Z_{o}(\max )$ & $0.98 \Omega$ & $0.11 \Omega$ \\
$L_{f}$ & $476 \mu \mathrm{H}$ & $95.2 \mu \mathrm{H}$ \\
$C_{f}$ & $0.085 \mathrm{~F}$ & $0.425 \mathrm{~F}$ \\
$R_{s l}$ & $3.4 \mathrm{~m} \Omega$ & $1.3 \mathrm{~m} \Omega$ \\
$R_{s c}$ & $2.2 \mathrm{~m} \Omega$ & $6.27 \mathrm{~m} \Omega$ \\
\hline
\end{tabular}

\section{Conclusion}

An ability to estimate ESR of electrolytic capacitors and winding resistance of inductor used in front end input filter, thereby enabling the prediction of possibility of a closedloop instability in a magnet power supply in the design stage itself, is of great practical importance. A way to estimate the capacitor ESR using datasheet values of commercially available capacitors and winding resistance of the filter inductor using empirical relationships is proposed in this paper that resulted in closed form expressions used to identify the stable operating region of the converter using simple spreadsheet calculations.

\section{Disclosure}

Rajul Lal Gour carried out M. Tech. project work at Power Supplies and Industrial Accelerator Division of Raja Ramanna Centre for Advanced Technology, Indore, during December 2015 to June 2016.

\section{Competing Interests}

The authors declare that they have no competing interests.

\section{References}

[1] P. J. Bryant, "Performance requirements for accelerators," in Proceedings of the Power Converters for Particle Accelerators, Hyatt Conference, Montreux, Switzerland, March 1990. 
[2] A. Elkiær, C. Nielsen, A. Jensen, and C. Sørensen, "Low voltage high current SCR controlled magnet power supply," in Proceedings of the 2nd International Particle Accelerator Conference (IPAC '11), Kyoto, Japan, 2011.

[3] X. Qi and Z. Xu, Resonant Magnet Power Supply System for the Rapid Cycle Synchrotron of Chinese Spallation Neutron Source, APAC Proceedings, Gyeongju, Korea, 2004.

[4] F. Long, "Status and trends in magnet power converter technology for accelerator," in Proceedings of the 5th International Particle Accelerator Conference (IPAC '14), Dresden, Germany, 2014.

[5] J.-C. Huang, Y.-S. Wong, and K.-B. Liu, "Improvement of output current characteristics for BIRA MCOR30 correction magnet power supply," in Proceedings of the IPAC, San Sebastian, Spain, September 2011.

[6] Y. Huaihai, Z. Zhongzu, G. Yaling et al., "A design of switch magnet power supply," in Proceedings of the 19th International Conference on Cyclotrons and Their Applications (CYCLOTRONS '10), Institute of Modern Physics, Chinese Academy of Science, Lanzhou, China, September 2010.

[7] S. C. Kim, S. H. Ahn, J. C. Yoon, J. M. Kim, C. D. Park, and K. R. Kim, "Magnet power supplies performance at PSL-II storage ring," in Proceedings of the IPAC, Pohang, Korea, 2016.

[8] S. H. Jeong, K.-H. Park, H. S. Suh et al., "Status of the fabrication of PAL-XFEL magnet power supplies," in Proceedings of the FEL, Daejeon Korea, 2015.

[9] R. D. Middlebrook and S. Cuk, Advances in Switched-Mode Power Conversion, Volume I and II, TESLAco, 2nd edition, 1983.

[10] R. W. Ercikson and D. Maksimovic, Fundamentals of Power Electronics, Springer, Berlin, Germany, 2nd edition, 2001.

[11] C. W. T. McLyman, Transformer and Inductor Handbook, PHI, 2nd edition, 2003.

[12] N. Mohan, T. M. Undeland, and W. P. Robbins, Power Electronics Converter, Application and Design, John Wiley \& Sons, New York, NY, USA, 3rd edition, 2002. 


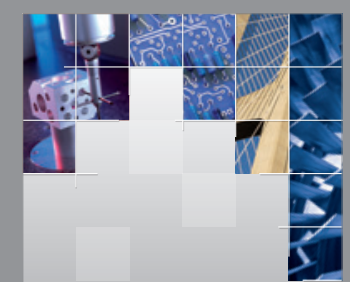

\section{Enfincering}
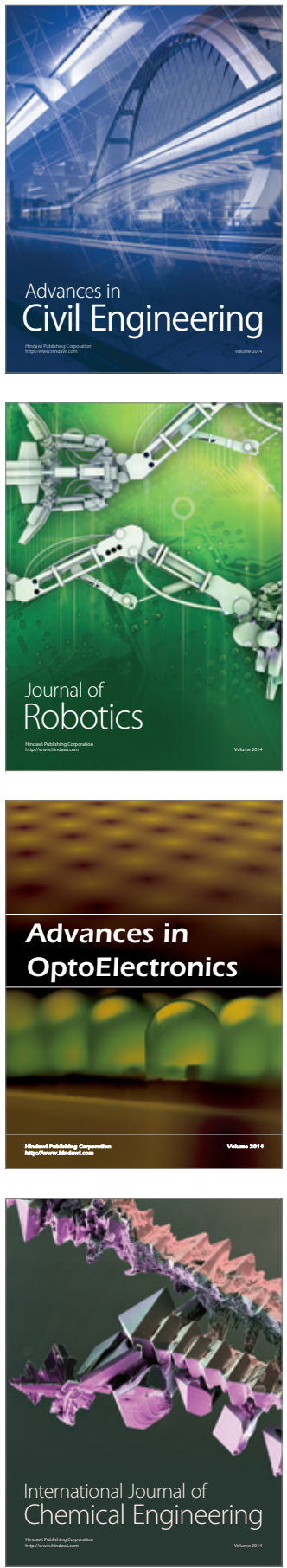

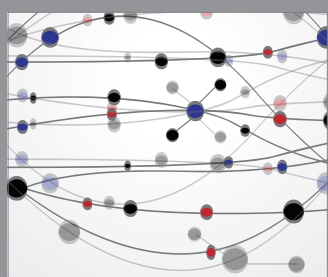

The Scientific World Journal

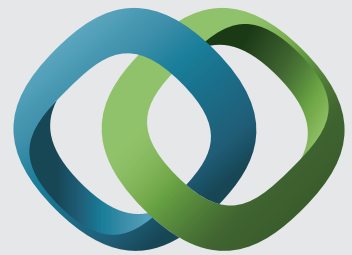

\section{Hindawi}

Submit your manuscripts at

http://www.hindawi.com
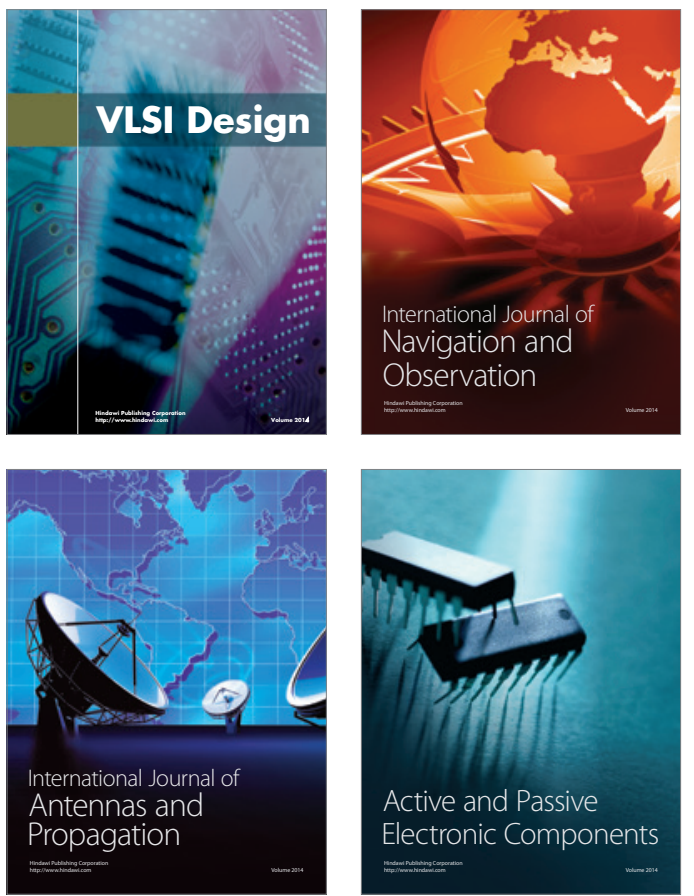
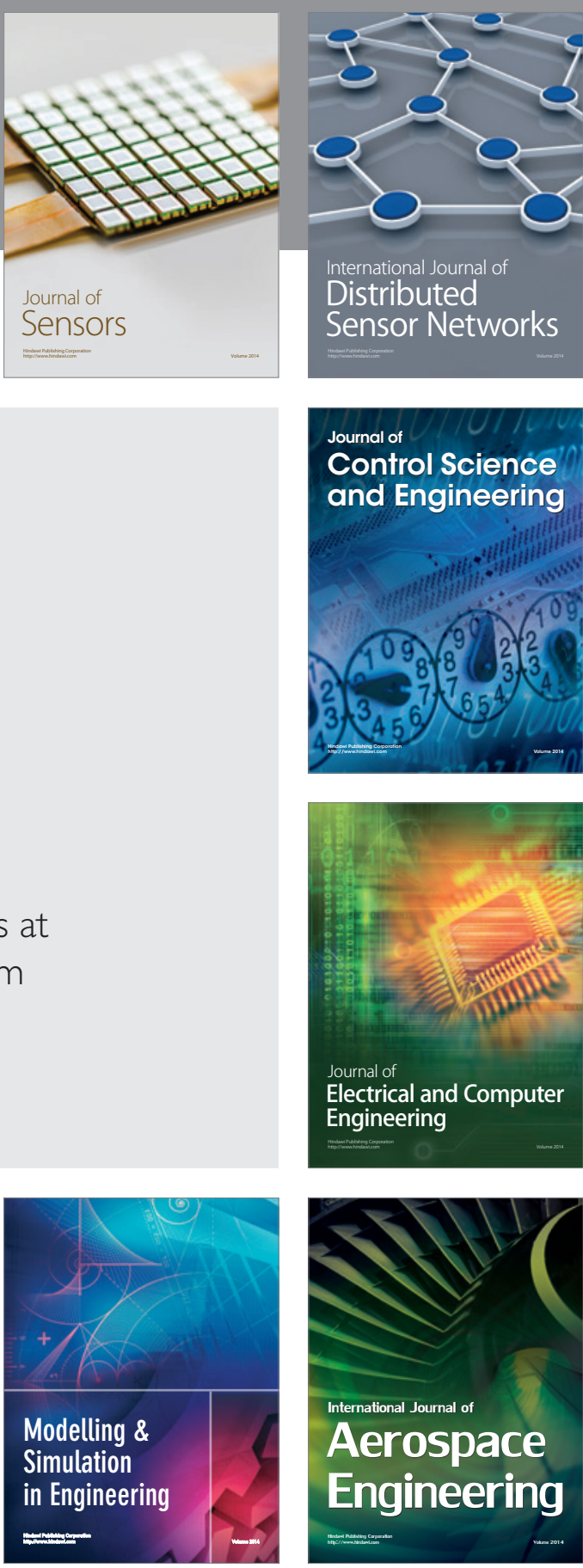

International Journal of

Distributed

Sensor Networks

Journal of

Control Science

and Engineering
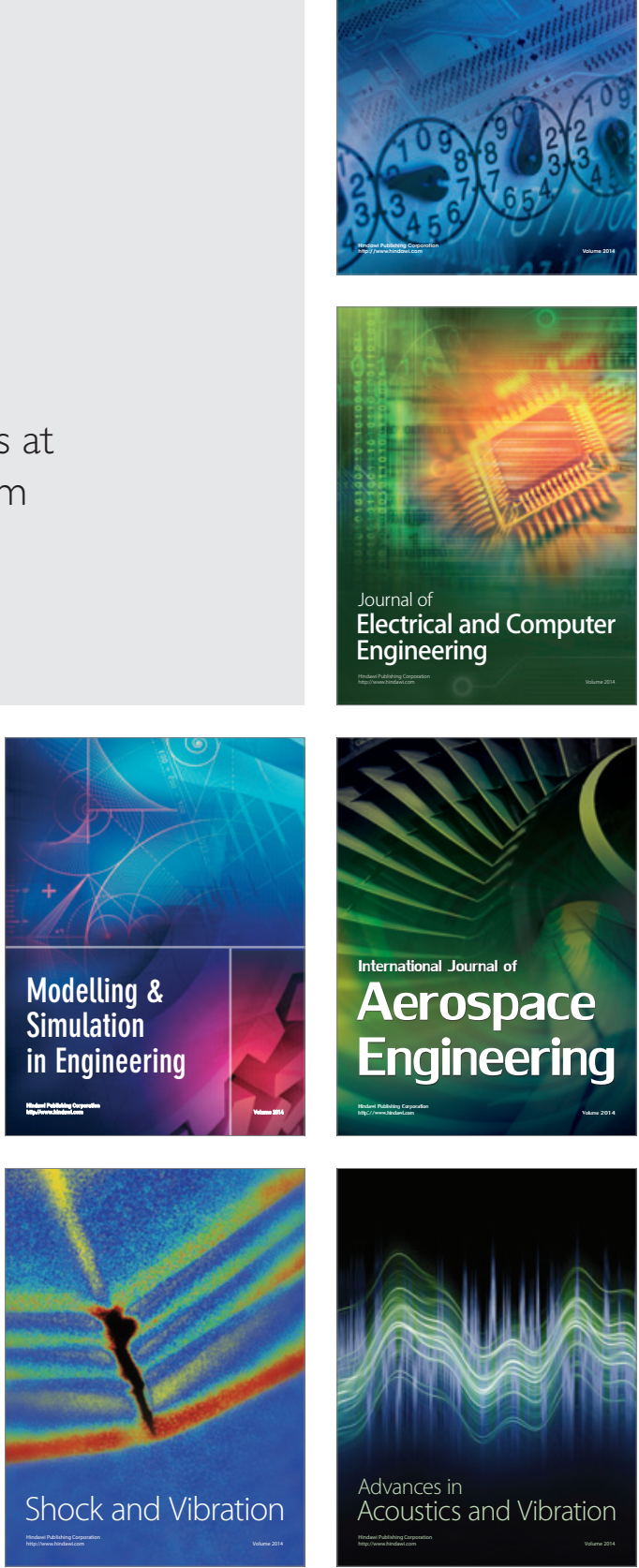